\title{
Sainte-Marie de Pleine-Selve en Gironde : nouvelles recherches sur une abbaye méconnue de l'ordre de Prémontré
}

Juliette Masson

\author{
(2) OpenEdition \\ Journals \\ Édition électronique \\ URL : https://journals.openedition.org/cem/13271 \\ DOI : 10.4000/cem. 13271 \\ ISSN : 1954-3093 \\ Éditeur \\ Centre d'études médiévales Saint-Germain d'Auxerre
}

Référence électronique

Juliette Masson, « Sainte-Marie de Pleine-Selve en Gironde : nouvelles recherches sur une abbaye méconnue de l'ordre de Prémontré », Bulletin du centre d'études médiévales d'Auxerre / BUCEMA [En ligne], 17.2 | 2013, mis en ligne le 10 décembre 2013, consulté le 22 septembre 2022. URL : http:// journals.openedition.org/cem/13271; DOI : https://doi.org/10.4000/cem.13271

Ce document a été généré automatiquement le 22 septembre 2022.

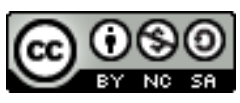

Creative Commons - Attribution - Pas d'Utilisation Commerciale - Partage dans les Mêmes Conditions 4.0 International - CC BY-NC-SA 4.0

https://creativecommons.org/licenses/by-nc-sa/4.0/ 


\title{
Sainte-Marie de Pleine-Selve en Gironde : nouvelles recherches sur une abbaye méconnue de l'ordre de Prémontré
}

\author{
Juliette Masson
}

L'opération archéologique sur le site de l'abbaye de Pleine-Selve ${ }^{1}$ a été programmée dans le cadre d'un doctorat portant sur les fondations liées à l'archevêque de Bordeaux Geoffroy du Loroux (1136-1158) ${ }^{2}$. Seuls subsistent aujourd'hui des vestiges de l'église abbatiale, les bâtiments conventuels ayant totalement disparu. Deux sessions ont été menées en 2007 et en 2008, financées par

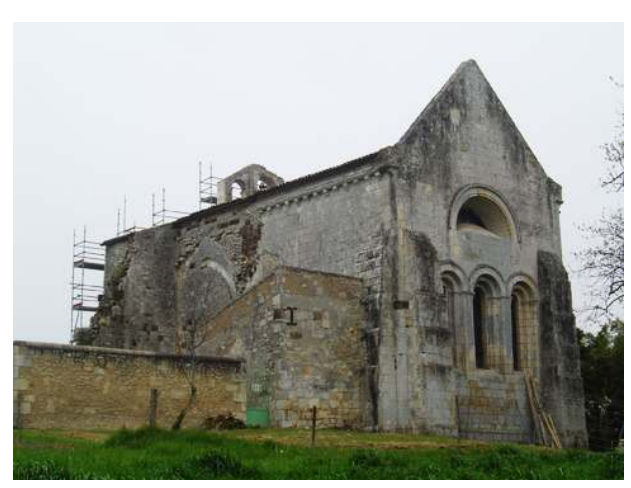
le Service régional de l'archéologie d'Aquitaine et le Conseil général de Gironde, pour conduire une étude archéologique de la construction de l'abbatiale et évaluer les vestiges enfouis des annexes par une prospection électrique. Le rapport de l'opération sur cet édifice, jamais étudié intégralement auparavant, a permis de proposer une nouvelle lecture de sa mise en œuvre, avec des hypothèses différentes de celles proposées par J.-A. Brutails ${ }^{3}$.

\section{Une fondation de Geoffroy du Loroux?}

2 Si l'on se fie à l'historiographie relative à la communauté de Pleine-Selve, l'abbaye aurait été implantée dans le diocèse de Bordeaux par l'archevêque Geoffroy du Loroux vers 1145-1150. 
Considéré comme l'une des plus fortes personnalités réformatrices du $\mathrm{xII}^{\mathrm{e}}$ siècle, Geoffroy du Loroux est écolâtre à Angers de 1096 à 1107, date à laquelle on perd sa trace dans les sources ${ }^{4}$. On le retrouve vers 1130 à la tête de deux communautés érémitiques : Fontaine-le-Comte, au sud de Poitiers, et Sablonceaux, en Saintonge, à la suite de la donation de ces lieux par le duc d'Aquitaine Guillaume X. Vers 1130, il obtient l'autorisation de fonder, en l'honneur de la Vierge, l'abbaye de Saint-Pierre de l'Isle en Médoc ${ }^{5}$. Auteur de nombreux sermons, il entretient des relations privilégiées avec Bernard de Clairvaux, Pierre le Vénérable, le roi Louis VII et son conseiller Suger.

4 Aucun acte de fondation n'a été conservé pour l'abbaye de Pleine-Selve. L'attribution à Geoffroy $\mathrm{du}$ Loroux repose sur quelques lignes reportées dans la Gallia Christiana signalant que Pleine-Selve fut confiée aux prémontrés par l'archevêque Geoffroi, d'après les annales de l'ordre de Prémontré, gardées dans la bibliothèque de Saint-Jean d'Amiens ${ }^{6}$. Les autres archevêques de Bordeaux, nommés Geoffroy (I et II), occupent le siège épiscopal respectivement au $\mathrm{x}^{\mathrm{e}}$ et au XI $\mathrm{xl}^{\mathrm{e}}$ sìcle. Il n'y a pas d'autre archevêque ainsi nommé pour le Moyen Âge à Bordeaux et l'abbaye de prémontré est fondée par Norbert de Xanten en 1120. Ainsi, l'auteur de cette notice désigne bien Geoffroy du Loroux (Geoffroy III) comme instigateur de l'implantation de cette communauté fille de Prémontré dans le diocèse de Bordeaux. Or, on ne sait pas à quel(s) document(s) il se réfère. Il consulte au moins un document postérieur à 1120, date de la création de l'ordre de Prémontré, et au moins un autre (ou le même) ne pouvant pas être antérieur à 1136, date de l'accession au siège épiscopal de Geoffroy du Loroux. D'après la mention qui en est faite dans la notice, le ou les documents en question ont pu être consultés au sein de la bibliothèque Saint-Jean d'Amiens, parmi les annales de l'ordre de Prémontré, mais les recherches dans cette voie sont demeurées infructueuses.

5 L'action de l'archevêque en faveur de la communauté de Pleine-Selve est toutefois évoquée par un texte du XII ${ }^{\mathrm{e}}$ siècle. Le cartulaire de l'abbaye Saint-Étienne de Baignes conserve un acte de l'archevêque Hardouin (1160-1163), daté de 1163, relatif à un litige entre la dite abbaye et les chanoines de Pleine-Selve au sujet de l'église de SaintPalais ${ }^{7}$. Cette église avait été donnée par l'archevêque Arnaud Géraud de Cabanac aux moines de Baignes, puis aux chanoines de Pleine-Selve par Geoffroy du Loroux. Aucune date relative à ces donations n'est mentionnée. Soit l'archevêque dote l'abbaye lors de sa fondation, soit il confie l'église à l'établissement déjà implanté. Ce texte est le plus ancien acte connu mentionnant l'abbaye de Pleine-Selve et le seul à présenter une action de l'archevêque envers cette communauté. Aucune indication n'est faite sur l'observance, quelle ait été prémontrée ou augustinienne, au moment de cette dotation, mais les religieux y sont nommés canonici. Ainsi, ce texte de 1163, qui mentionne des défrichements où sont levées des dîmes novales et où l'on projette la mise en place d'un cimetière habité juxta ecclesiam de Plana Silva, révèle une implantation plutôt réussie, avec un lieu de culte déjà en place, antérieur ou en cours d'élévation ${ }^{8}$.

\section{Un vaste enclos abbatial dévoilé par des plans modernes}

6 Trois documents présentant un plan de l'abbaye révèlent un vaste enclos abbatial comprenant deux cloîtres, plusieurs cours et jardins, diverses annexes, le tout fortifié par une enceinte munie de tours et entouré partiellement d'un cours d'eau. Un premier 
plan fut remis par l'abbé Mestivier aux archives départementales de la Gironde en 1892 (fig. 1) ${ }^{9}$. Le second plan a été publié en 1982 dans l'article de Bernard Peyrous, où il cite M. Darricau comme détenteur du document, mais nous n'avons pas retrouvé sa trace (fig. 2) ${ }^{10}$. Enfin, le troisième plan apparaît en photocopie dans le dossier relatif à PleineSelve à la Conservation régionale des Monuments historiques (fig. 3) ${ }^{11}$. L'analyse de ces plans indique que les trois documents sont des copies modernes et contemporaines d'un document original, non retrouvé à ce jour ${ }^{12}$. Même s'il semble être lui-même une copie, le plan conservé dans le dossier des Monuments historiques semble bien être le document originel par rapport aux deux autres plans. La première copie de ce plan est celle publiée par B. Peyrous, et cette copie a ensuite été reproduite par l'abbé Mestivier. D'après l'estimation qu'en fait B. Peyrous, le document originel serait antérieur au dernier tiers du XVI ${ }^{\mathrm{e}}$ siècle.

Fig. 1 - Copie d'un plan de l'abbaye de Pleine-Selve remis par l'abbé Mestivier aux Archives départementales de la Gironde en 1892. Non coté (cl. AD Gironde, 2008).

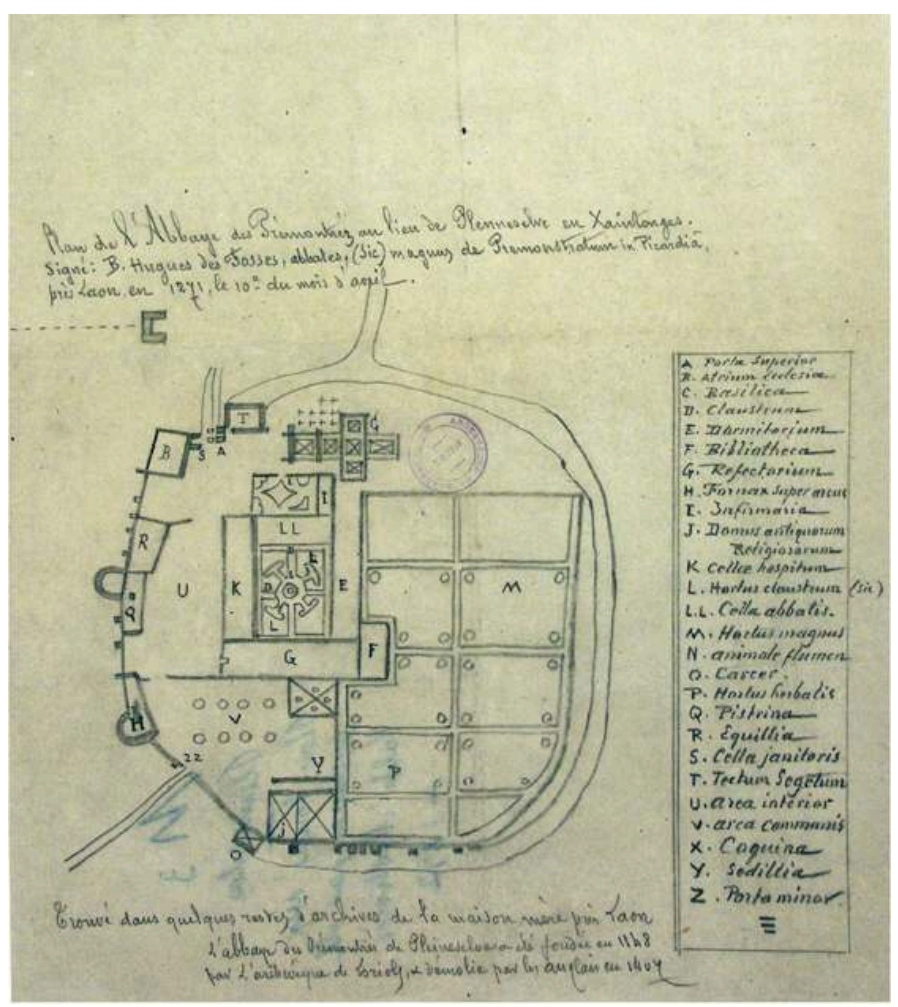


Fig. 2 - Plan de l'abbaye de Pleine-Selve publié dans B. PeYrous, «Les prémontrés de PleineSelve ", Cahiers du Vitrezais : abbayes et couvents du Blayais, Paris, 1982, p. 55-62. Ce document n'ayant pas été retrouvé.

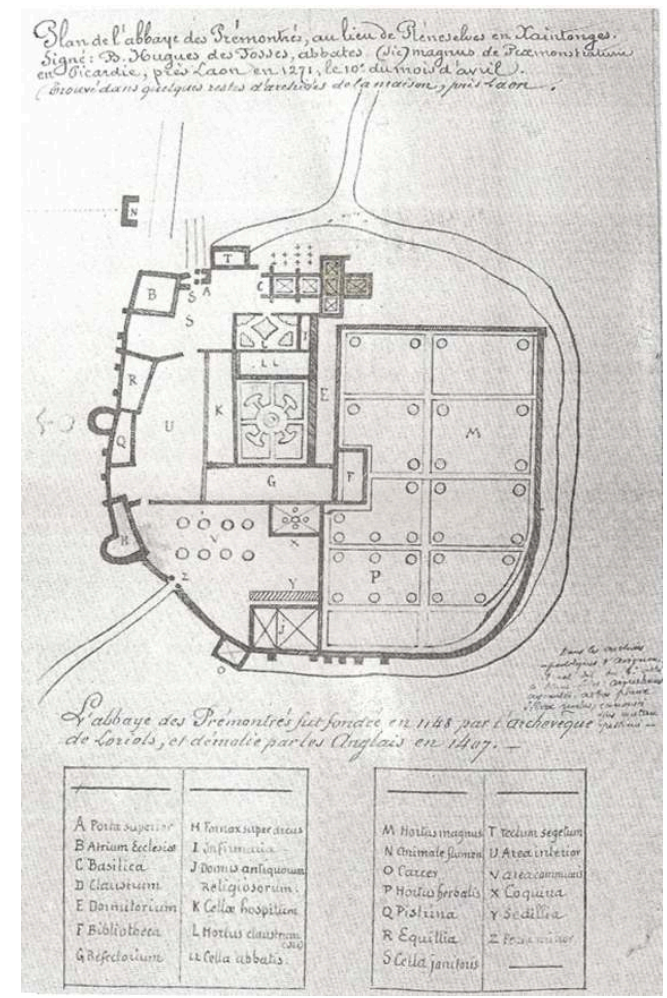

Fig. 3 - Plan de Pleine-Selve dans le dossier relatif à l'abbaye à la Conservation régionale des Monuments historiques (Gironde). C'est une photocopie papier d'une photographie argentique ; la source indiquée est la mairie de Pleine-Selve, où le document n'est plus conservé.

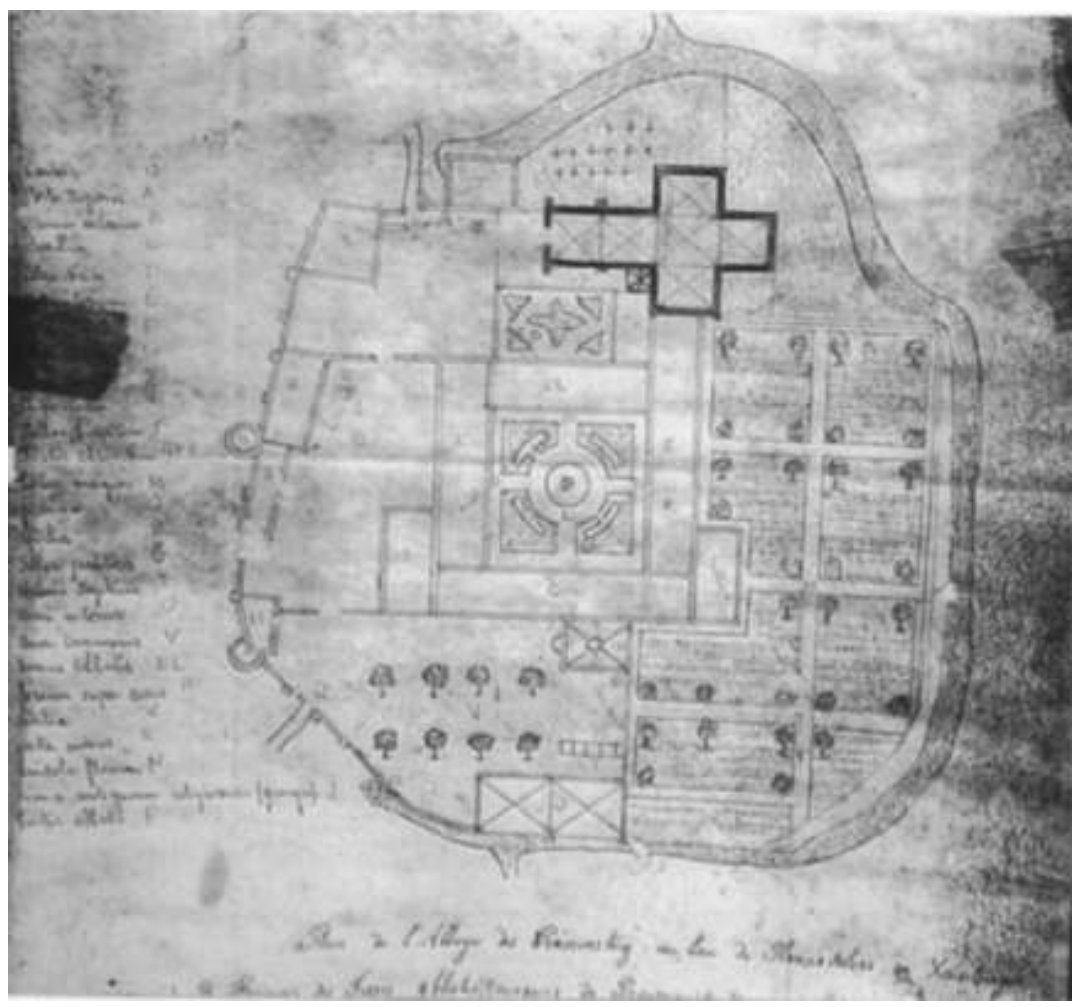


7 Ces plans présentent un enclos avec cinq espaces bien définis : l'église et les bâtiments autour du cloître au centre, des grands jardins à l'est, un espace précédant la porte majeure au nord et servant d'atrium pour l'église, un espace intérieur avec boulangerie et écurie à l'ouest, et un espace commun au sud ouvert sur l'extérieur par la porte mineure. Ne sont pas mentionnées certaines fonctions propres aux abbayes conservées ou dont le plan est connu, tel la salle capitulaire, la sacristie, le trésor, le chauffoir et le lavabo dans le cloître pour les lieux de vie régulière, ou encore la buanderie, un vivier, un moulin ou une forge pour les annexes. De même, aucun couloir de passage n'est indiqué, couloir fréquemment présent notamment pour lier le cloître et les jardins. Ces plans pourraient être soit la représentation d'un seul étage des bâtiments, mais les pièces désignées ne s'y prêtent pas, soit l'évocation d'un état ponctuel de l'abbaye.

Les deux cours $\mathrm{U}$ et $\mathrm{V}$ sont ouvertes sur l'extérieur et pouvaient être utilisées pour l'accueil des visiteurs. Ainsi les chanoines pouvaient continuer leur vie régulière sans être mélangés avec leurs hôtes de passage. Ces derniers pouvaient pénétrer dans l'enceinte au nord (porte A, espace B), gagner leur lieu d'accueil (bâtiment K, espace U) ou l'église, sans perturber les chanoines. Il est vraisemblable que la porte sud (mineure) ne soit destinée qu'aux religieux (convers) et que la porte nord (majeure) soit l'entrée effectivement destinée aux visiteurs. La circulation des visiteurs pourrait être ainsi assez perceptible. En revanche, la circulation des chanoines parait moins évidente à appréhender. Aucune ouverture n'est indiquée dans les différents bâtiments liés à la vie régulière. Ce déséquilibre dans les détails pourrait être un indice à prendre en compte pour comprendre la motivation de la commande du plan de l'abbaye et la fonction qui lui était destinée.

\section{Nouvelle lecture de la mise en œuvre de l'église abbatiale}

\section{État de la question avant l'étude menée en 2007-2008}

9 Les seuls vestiges de l'abbaye sont ceux de l'église encore en élévation, entre le cimetière actuel à l'ouest et des champs de vignes à l'entour (fig.4). Le plan actuel présente un chevet plat, la croisée du transept et le bras nord ouvert sur une chapelle orientée à fond plat (fig. 5). Les arcades sud et ouest de la croisée du transept ont été murées, fixant dans cet état un édifice très ruiné. Dans les parties hautes de la croisée, des traces révèlent le voûtement en coupoles sur pendentifs qui se prolongeait dans la nef disparue. L'unique travée du chevet est de plan quasiment carré, de côté égal à 6,75 m en moyenne (fig. 6). 
Fig. 4 - Photographie de l'actuelle façade occidentale de l'église abbatiale de Pleine-Selve : on peut observer les vestiges du pendentif de la coupole qui voûtait la première travée de la nef (cl. Juliette Masson, 2007).

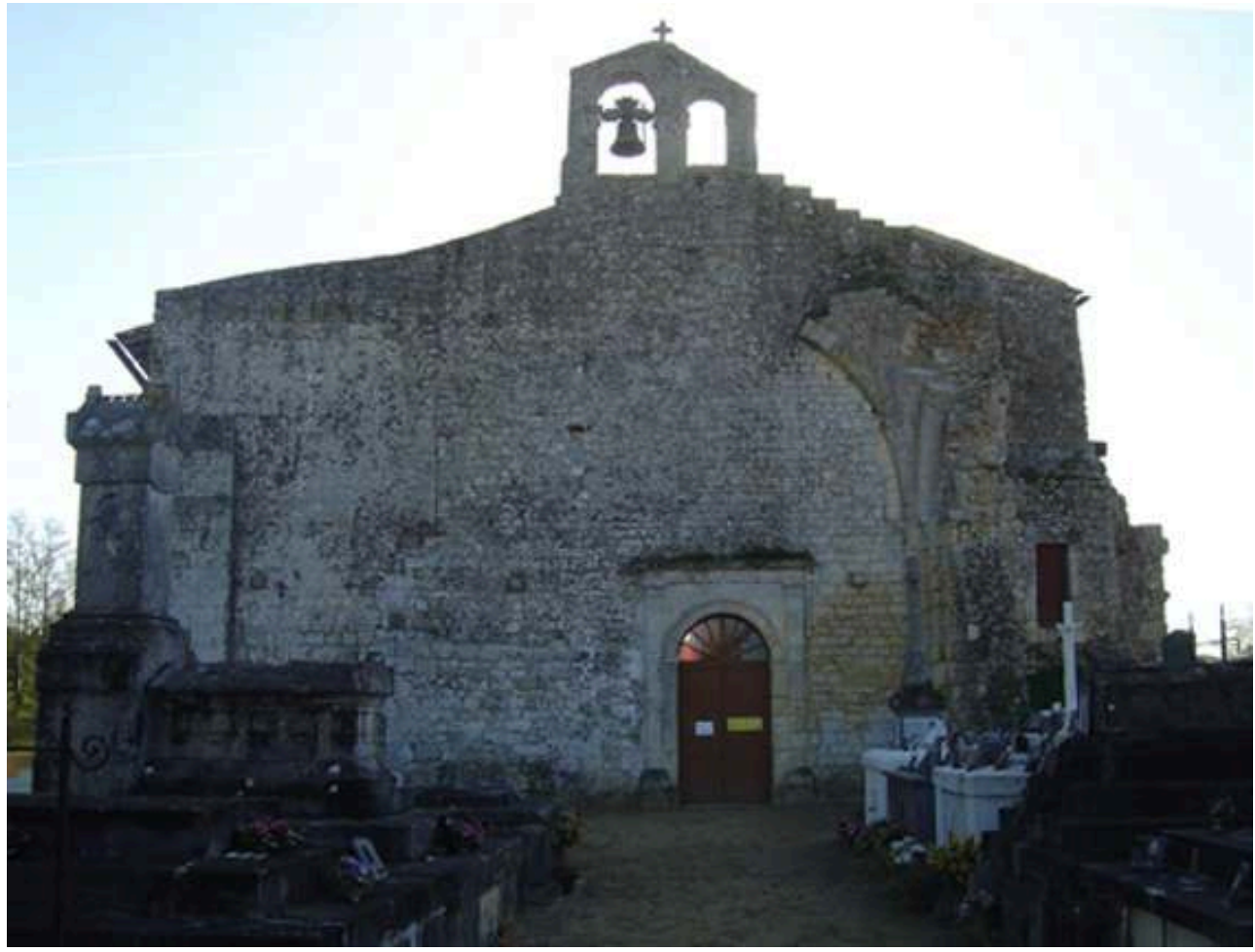

Fig. 5. Plan des vestiges de l'église abbatiale de Pleine-Selve (données : Michel Goutal (2004) et Juliette Masson (2007-2008) ; DAO : Juliette Masson, 2008).

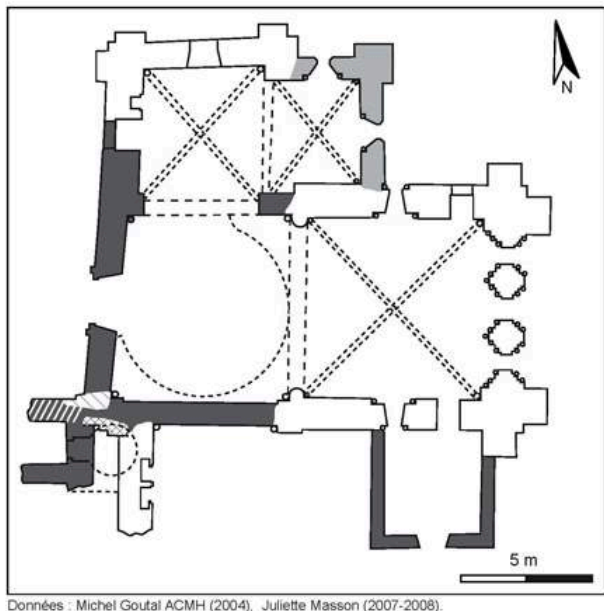

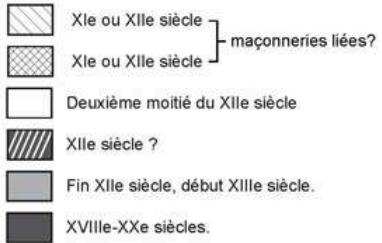


Fig. 6 - Photographie intérieure du chevet de l'abbatiale de Pleine-Selve (cl. Juliette Masson, 2008).

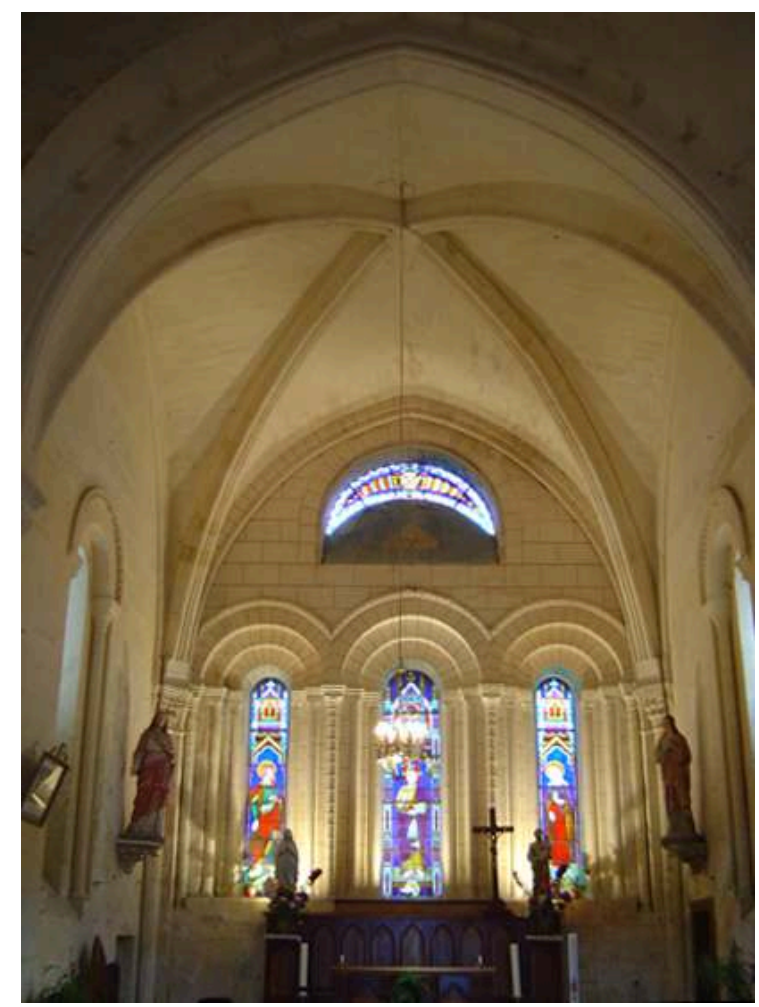

10 La croisée du transept présente approximativement les mêmes dimensions. Le bras nord du transept a un plan presque carré, mais plus petit, avec un côté de 4,40 m. De plan rectangulaire, la chapelle est large de 3,80 m et profonde de près de $2,70 \mathrm{~m}$. Le bras sud du transept, ruiné, a conservé une longueur de 4,30 mètres pour son élévation occidentale.

11 D'après les mesures faites sur les vestiges de la nef, qui affleuraient encore au début du $\mathrm{xx}^{\mathrm{e}}$ siècle, elle devait avoir une longueur proche des trente mètres. Selon ses observations, J.-A. Brutails estimait la longueur de l'église à 44 mètres et la largeur au niveau du transept de $17,40 \mathrm{~m}$, ce qui correspond aux vestiges actuels ${ }^{13}$. Il considérait l'édifice comme le témoin d'un même programme, avec une nef qui présentait vraisemblablement trois travées, toutes trois voûtées d'une coupole circulaire sur pendentifs. Cependant, sur les trois plans connus de l'abbaye, la nef est figurée avec seulement deux travées. L'analyse des élévations menée en 2007 et 2008 a révélé des indices propices à une nouvelle lecture de la mise en œuvre de l'église.

\section{Nouvelle analyse de la construction}

12 L'étude de terrain a eu pour objectif l'étude de l'église mais aussi la recherche d'éventuels vestiges enfouis des bâtiments conventuels. Afin de déceler l'emplacement de ces derniers et de faire un parallèle avec les plans connus de l'abbaye, une prospection électrique a été menée aux abords de l'église et du cimetière actuel. Le principe de la prospection électrique est de créer un champ électrique artificiel et de mesurer ensuite la résistivité du sol, le signal étant stoppé par des structures en pierre ou accéléré par l'humidité de fosses au comblement plus meuble que l'encaissant. Même si quelques structures sont apparues, cette tentative n'a malheureusement pas 
donné des résultats satisfaisants (fig.7). En effet, il apparaît que le sol a été très remanié et plusieurs des résistances mesurées peuvent être liées aux activités agricoles voisines. Seuls des sondages ponctuels, dont l'emplacement est suggéré par les zones de forte résistivité, pourraient apporter des éléments supplémentaires pour interpréter au mieux ces résultats.

Fig. 7 - Résultats de la prospection électrique réalisée autour de l'église abbatiale, superposés au levé topographique et au plan de l'abbé Mestivier. Prospection réalisée par Marion Druez, ULR-Valor (La Rochelle), 2008.

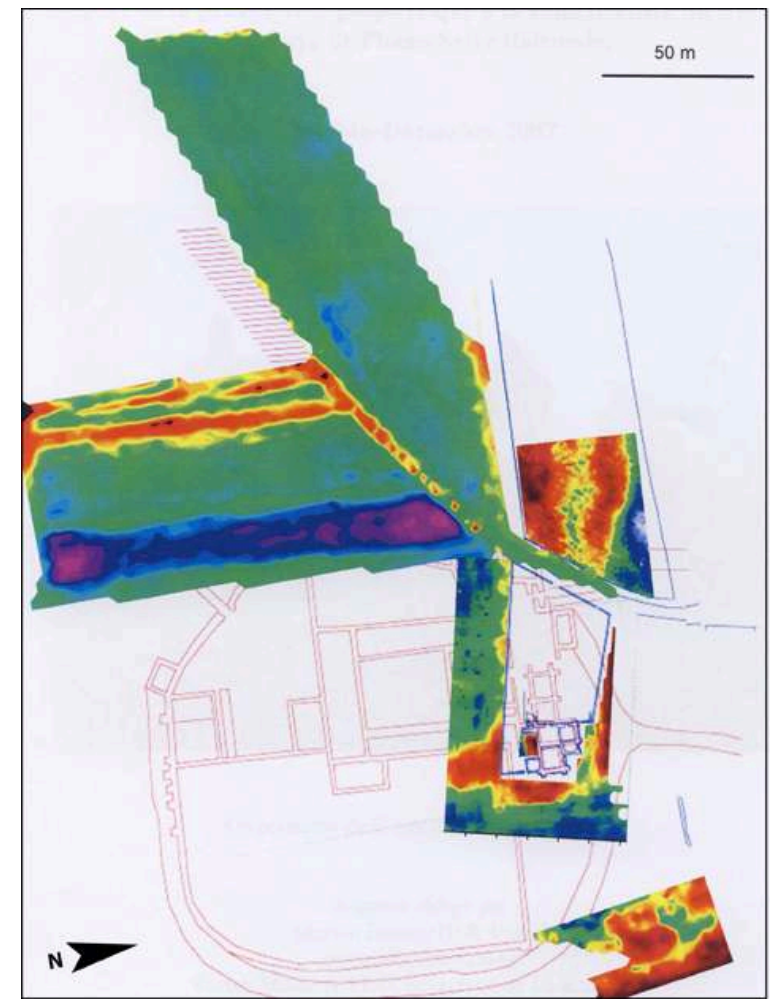

13 En revanche, pour l'église abbatiale, la lecture méticuleuse de chaque élévation a mis en évidence plusieurs éléments qui permettent de comprendre la mise en œuvre de l'édifice. La mesure des hauteurs d'assises dans les élévations du chevet et des deux bras du transept révèle que ces trois espaces, chevet, bras nord et bras sud, ont été élevés en même temps et non pas l'un après l'autre, et ce au moins jusqu'en partie haute des murs. Le même appareil se retrouve dans la partie basse des élévations de ces trois espaces et plusieurs assises bien plus étroites que les autres sont observables à des hauteurs quasi équivalentes sur toutes les élévations. Enfin, un même appareil plus régulier et plus soigné est visible dans la partie supérieure de ces mêmes élévations. L'analyse du calcaire et du mortier utilisés, par observation directe, confirme ces observations.

14 À la jonction du transept et de la nef, d'autres nouveaux indices ont été repérés, utiles à la compréhension de la mise en œuvre. À l'angle entre le bras sud du transept et de la nef, une tourelle d'escalier subsiste encore, portant les traces de multiples reprises et menant à un clocher-mur installé sur l'actuelle façade occidentale. L'escalier donne accès aussi à une vis antérieure, en partie conservée, à la jonction entre le bras et la croisée du transept. Cette ancienne vis menait plus haut, mais sa destination primitive est perdue. Cette tour, dans son état actuel, est postérieure à la fermeture de l'église 
ruinée au niveau de la croisée du transept. Un couloir voûté sert de soubassement à l'actuelle tourelle et aboutit contre un parement en pierre de taille, avec un contrefort en partie visible, à la jonction entre la nef et la croisée. Cependant ce parement ne fonctionne pas avec les vestiges du transept et, en outre, les bases de la tourelle viennent se poser contre ce même parement. De plus, les vestiges de la travée occidentale de la nef, conservés dans l'actuelle façade, présente les éléments d'un pendentif reposant sur un support bien différent des autres supports observés dans l'église, notamment par les chapiteaux lisses qui le coiffent. Le support de l'angle sudouest de la croisée est visiblement greffé sur ce pilier à chapiteaux lisses à la jonction entre la croisée et la nef. L'ensemble du transept et du chevet plat serait donc un seul et même programme prolongeant une nef antérieure. Les vestiges du parement et d'un contrefort observés sous la tourelle d'escalier appartiendraient à cette nef voûtée de coupoles, mais il faudrait démanteler la tour pour s'en assurer.

Les éléments $\mathrm{du} \mathrm{XII}^{\mathrm{e}}$ siècle conservés au sein de l'église présenteraient donc non pas un seul et même projet, comme le pensait J.-A. Brutails, mais deux projets distincts, pas forcément éloignés dans le temps, mais témoins d'un édifice antérieur non décelé auparavant. Le programme de la nef à file de coupoles est antérieur à l'église au chevet à fond plat : il appartiendrait à un premier projet modifié en cours de route ou à un édifice pour lequel les parties orientales et le transept auraient été remplacés.

Des reprises sont réalisées ensuite dès la fin du XII ${ }^{e}$ siècle et au début du XIII ${ }^{e}$ siècle. Sur chaque bras du transept une chapelle plus vaste est mise en place, à fond plat, greffée aux murs nord et sud du chevet. La chapelle nord est la première construite et est toujours en place aujourd'hui. Elle semble avoir été voûtée dès sa construction, et c'est probablement à cette occasion que d'autres parties de l'édifice reçoivent des ogives. La construction de la chapelle sur le bras sud est lancée après celle de la chapelle nord. Le mur oriental du bras sud est démantelé à cette fin: on observe encore au début du $\mathrm{xx}^{\mathrm{e}}$ siècle les traces d'arrachement situées à son emplacement ${ }^{14}$. Le parement sud du chevet est remanié dans sa partie haute et taillé pour y greffer un arc brisé (fig. 8). Les murs de la chapelle ont pu être élevés, mais le voûtement ne semble pas avoir été achevé, même si des indices de toiture sont visibles (fig. 9). 
Fig. 8 - Photographie de l'élévation sud de l'église abbatiale de Pleine-Selve : on peut observer les vestiges du transept et les traces des travaux pour réaliser une chapelle (cl. Juliette Masson, 2008).

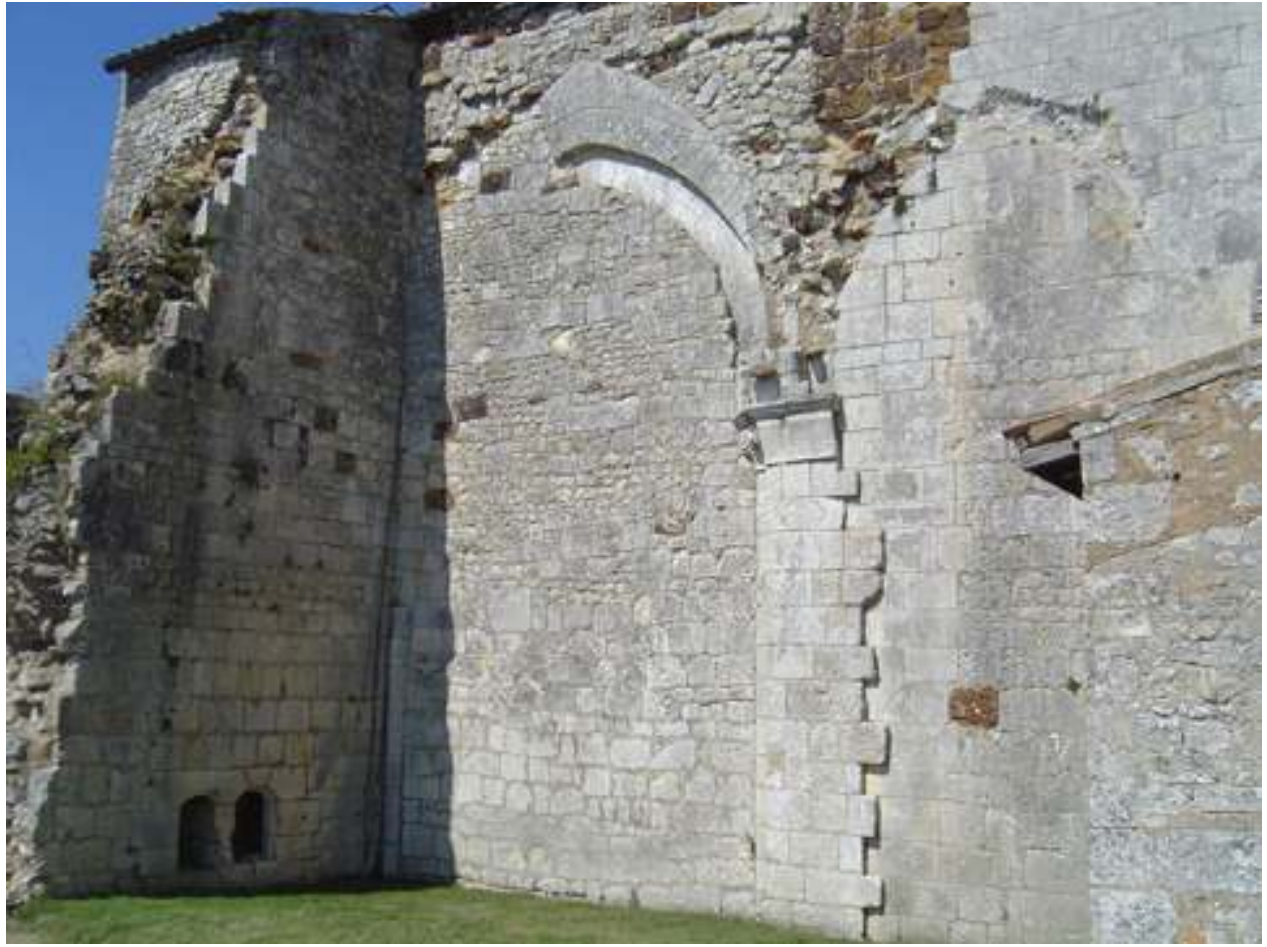

Fig. 9 - Relevé et étude de la construction de l'élévation sud de l'église abbatiale de Pleine-Selve (données : Michel Goutal, ACMH (2004) et Juliette Masson (2007-2008) ; DAO : Juliette Masson, 2008).

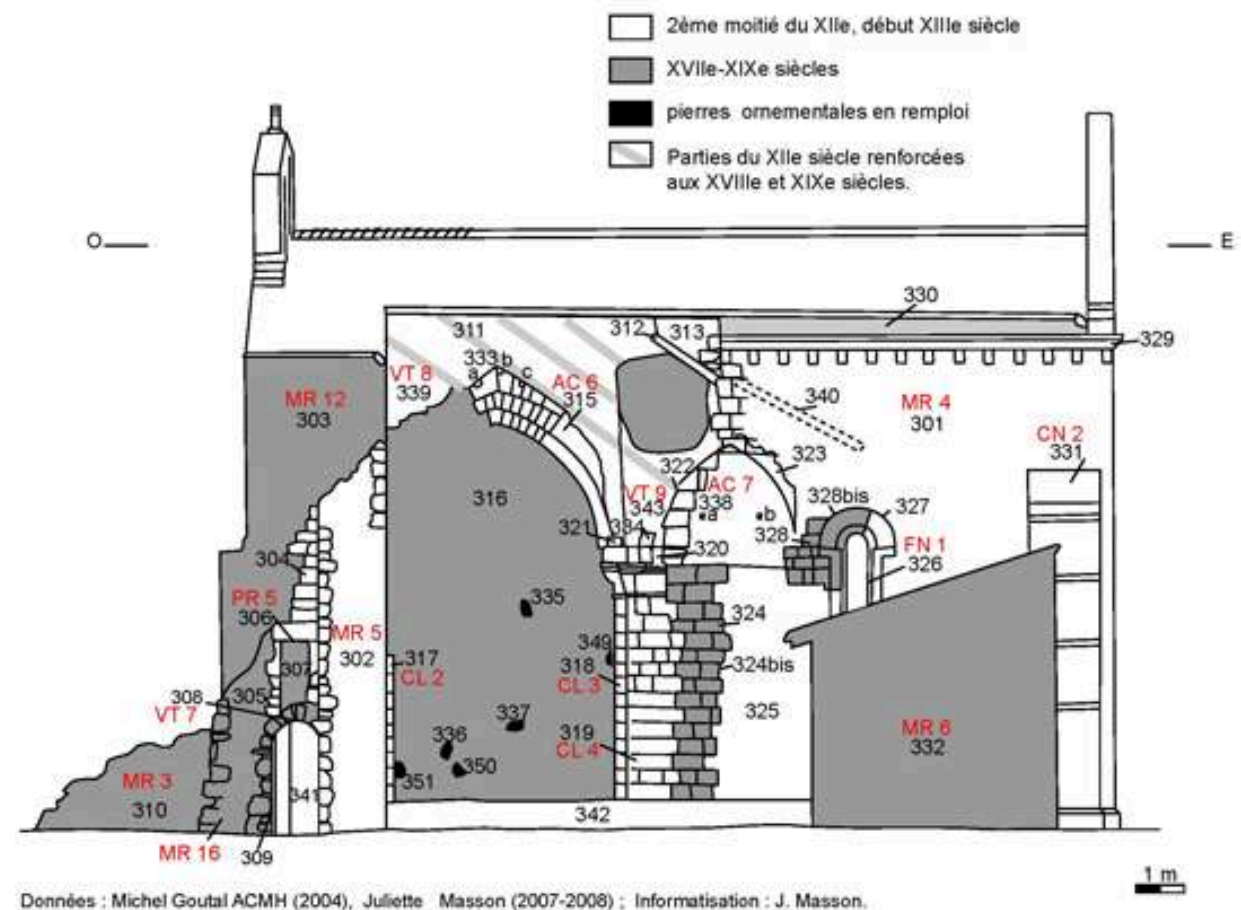

Bulletin du centre d'études médiévales d'Auxerre | BUCEMA, 17.2 | 2013 


\section{Pleine-Selve face aux fondations attestées de Geoffroy du Loroux}

L'étude menée sur les abbayes de Fontaine-le-Comte (Vienne), Sablonceaux (CharenteMaritime) et Saint-Pierre de l'Isle (Gironde) a mis en évidence l'adoption d'un programme architectural identique pour les trois abbatiales (fig. 10).

Fig. 10 - Table comparative du plan des abbatiales liées à Geoffroy du Loroux.

Une nef à trois vaisseaux, un transept saillant muni d'absidioles orientées et une vaste abside pour le chevet, un décor très sobre et un couvrement en coupoles sur pendentifs et en berceaux brisés constituent les principales caractéristiques de ce programme. L'attribution à Geoffroy du Loroux de leur fondation, entre 1130 et 1136, est bien attestée par des actes conservés. Même si Pleine-Selve présente des vestiges d'une nef antérieure, celle-ci n'a pas les mêmes caractéristiques que la nef répétée dans les trois abbatiales implantées par le prélat: la nef de Pleine-Selve est plus étroite et la composition des supports diffère nettement. Il en est de même pour les parties orientales encore en place, datables de surcroît de la fin du XII ${ }^{\mathrm{e}}$ siècle. Il ne faut pas en déduire pour autant que Pleine-Selve ne peut pas être une fondation de l'archevêque seulement parce que son programme est distinct des trois autres, car elle pourrait tout autant refléter un changement de son investissement dans la commande. Toutefois elle reste à part dans ce modeste corpus lié au fondateur.

Le lien entre Geoffroy du Loroux et la fondation de l'abbaye de Pleine-Selve demeure, mais il reste néanmoins à nuancer. À ce jour, le contexte de fondation de Pleine-Selve reste obscur par bien des aspects, tout comme l'implantation en ces lieux des chanoines de Prémontré. En revanche, l'étude architecturale met en évidence un édifice antérieur, principalement caractérisé par son voûtement en file de coupoles sur pendentifs. L'étude documentaire a, en outre, fait ressortir des plans d'un grand enclos abbatial révélant le potentiel de ce site, qui, même si les textes restent muets quant à la place éventuelle de cette fondation dans la politique de l'archevêque, restera lié à la personnalité du grand réformateur que fut Geoffroy du Loroux en Aquitaine au $\mathrm{XII}^{\mathrm{e}}$ siècle. Il ressort de l'étude l'intérêt à porter à ces églises rurales, souvent délaissées, tant elles apportent des indices utiles à la connaissance de l'évolution des formes et de la mise en œuvre dans l'architecture au Moyen Âge.

Reçu : 31 août 2013 - Accepté : 4 décembre 2013

\section{NOTES}

1. Pleine-Selve (dép. Gironde, arr. Blaye, cant. Saint-Ciers-sur-Gironde).

2. J. MASSON, «Geoffroi du Loroux et l'architecture religieuse en Aquitaine au XII siècle », Bulletin du centre d'études médiévales d'Auxerre | BUCEMA [En ligne], 17.1 | 2013, mis en ligne le 19 juin 2013. URL : http://cem.revues.org/12985.

3. J.-A. BRUTAILS, Les vieilles églises de la Gironde, Paris, 1912, p. 82-84 ; R. BIRON, « Notice historique sur Sainte-Marie-Madeleine de Pleine-Selve ", Revue historique de Bordeaux et la Gironde, 26 (1934), p. 97-102. 
4. J.-H. FOULON, « Un représentant de la spiritualité canoniale au XII ${ }^{\mathrm{e}}$ siècle ? Geoffroy du Loroux ( $†$ 1158) », in M. PARISSE (dir.), Les chanoines réguliers, émergence et expansion (XI ${ }^{e}$-XIII ${ }^{e}$ siècles), SaintÉtienne, 2009, p. 71-115.

5. Fontaine-le-Comte (dép. Vienne, arr. Poitiers, cant. Poitiers-5), Sablonceaux (dép. CharenteMaritime, arr. Saintes, cant. Saujon) et Saint-Pierre de l'Isle (dép. Gironde, arr. et cant. LesparreMédoc, com. d'Ordonnac).

6. Gallia Christiana, Paris, 1751, t. 2, col. 892 : Plana-Silva filia Praemonstrati, nunc sine ullo canon. Praemonstr. conditur a Gaufredo ejusdem sedis archipraesule, ut fidem faciunt annales MSS. ordinis Praemonstra. ; in bibliotheca Sancti Johannis Ambianensis asservati ; ex quibus etiam nuda haec abbatum nomina eruimus.

7. Abbé CHOLET, Cartulaire de Saint-Étienne de Baignes, Niort, 1868, p. 45.

8. Abbé CHоlet, Cartulaire..., ibid. : Canonici de Plana Silva, quibus posterius illa ecclesia data fuerat, in manu nostra eam in capitulo suo refutaverunt, eam in pace dimittentes ecclesie de Beania, que eam de donatione bone memorie A. (Arnaudi) archiepiscopi reclamabat. Illa autem ecclesie dimissio, sub hac pactionis forma facta fuit, ut canonicis illam novalibus que sunt juxta ecclesiam de Plana Silva fieret cimiterium, ubi mansionem haberent homines sui, verum homines sui, de novalibus que sunt citra aquam mansionarii, nullo jure parrochiali ecclesie sancti Palladii tenerentur, neque parrochiani Sancti Palladii ad novalia illa, neque ad capellam, si ibi fieret, admitterentur [...].

9. Archives départementales de la Gironde, dessin au crayon sur calque, non coté.

10. B. Peyrous, «Les prémontrés de Pleine-Selve », Cahiers du Vitrezais: abbayes et couvents du Blayais, Paris, 1982, p. 55-62. Le plan est publié dans l'article de B. Peyrous où il signale que, en 1873 , le plan était en la possession de M. Manizan, puis il cite M. Darricau comme détenteur du document.

11. Ce document est consultable à la Conservation régionale des Monuments historiques. Ce dossier est une étude préalable menée en 2005 en vue de restaurations entreprises dès 2009. C'est une photocopie papier d'une photographie (argentique); la source indiquée est la Mairie de Pleine-Selve, où le document n'est pas (ou plus) conservé.

12. Pour une étude comparative approfondie des trois plans, voir J. MASSON, Geoffroi du Loroux et l'architecture religieuse en Aquitaine au XII siècle, p. 574-588 [thèse en ligne: http://tel.archivesouvertes.fr/tel-00735961/].

13. J.-A. BRUTAILS, Les vieilles..., op. cit., p. 82-84.

14. J.-A. B RUTAILS, Les vieilles..., ibid., p. 83 : les traces d'arrachement sur l'élévation sud sont visibles sur une photographie publiée par l'auteur dans sa notice.

INDEX

Mots-clés : abbaye, architecture, archéologie du bâti, archevêque, chanoine, commanditaire, Geoffroy du Loroux, prospection géophysique, réforme

Index géographique : France/Bordeaux, France/Fontaine-le-Comte, France/Pleine-Selve, France/Prémontré, France/Sablonceaux, France/Saint-Pierre de l'Isle 
AUTEUR

JULIETTE MASSON

Université de Bordeaux 3, UMR 5607 